\title{
Use of Session Rating of Perceived Exertion for Monitoring Resistance Exercise in Children who are Overweight or Obese
}

\author{
Michael R. McGuigan, Abdulaziz Al Dayel, David Tod, \\ Carl Foster, Robert U. Newton, and Simone Pettigrew
}

\begin{abstract}
The purpose of this study was to investigate the use of the OMNI Resistance Exercise scale (OMNI-RES) for monitoring the intensity of different modes of resistance training in children who are overweight or obese. Sixty-one children (mean age $=9.7 \pm 1.4$ years) performed three resistance training sessions every week for 4 weeks. Each session consisted of three sets of 3-15 repetitions of eight different resistance exercises. OMNI-RES RPE measures (0-10) were obtained following each set and following the end of the exercise session. There was a significant difference between average RPE $(1.68 \pm 0.61)$ and Session RPE (3.10 $\pm 1.18)$ during the 4 weeks of training $(p<.05)$. There was no significant change in session RPE over the 4 weeks of training. The correlation coefficient between average and session RPE values was significant $(r=.88, p<.05)$. The findings of the current study indicate that the RPE values are higher when OMNI-RES measures are obtained following the whole training session than when obtained following every single set of exercise. This suggests that in children the session RPE provides different information to the average RPE across the entire session.
\end{abstract}

One problem facing strength and conditioning professionals and researchers is how to monitor the intensity of different modes and phases of resistance training, particularly in different populations. The session RPE has been proposed as a method for monitoring the intensity of the global exertion $(6,7)$, including resistance training $(2,17,18)$. The session RPE allows the participant to provide a global rating of how hard the exercise session was, rather than reporting a series of RPE measures throughout the session (6). The goal of the session RPE is to encourage the participant to view the training session globally and to reduce the need for periodic reporting of exertional cues during the exercise bout. Although validated in healthy

\footnotetext{
McGuigan and Newton are with the Vario Health Institute, School of Exercise, Biomedical and Health Sciences, Edith Cowan University, Joondalup, WA, Australia. Al Dayel is with the School of Exercise, Biomedical and Health Sciences, Edith Cowan University, Joondalup, WA, Australia. Tod is with the Dept. of Sport and Exercise Science, Aberystwyth University, Australia. Foster is with Dept. of Exercise and Sports Science, University of Wisconsin-La Crosse, La Crosse, WI. Pettigrew is with the University of Western Australia, Australia.
} 
adult populations $(6,7)$, whether this method can be used effectively in different populations such as children and the elderly has yet to be confirmed. As children are psychologically less mature than adults, they might have difficulty using any type of RPE scale to provide a global rating of the exercise session.

The Borg 15-Category scale has been widely used to evaluate the intensity of exertional perceptions in a variety of different settings (14). The application has mainly been used during aerobic exercise, but recently some investigators have studied its use with resistance training $(11,12)$. Several studies have demonstrated the Borg scale to be an effective method of quantifying resistance training intensity $(11,12)$. Most studies have used college-aged participants, however. The OMNI Perceived Exertion Scale for Resistance Exercise (OMNI-RES) was developed by Robertson et al. (16) to be more expressive of exercise intensity and to be more comprehensible, particularly for children. Three descriptors are used in this scale: six verbal phrases, four mode pictorials, and numerical figures from 0 to 10 distributed along an inclined line. Its use has previously been validated in normal weight children (15). Faigenbaum et al. (4) have established the validity and reliability of an 11-point numerical scale with five pictures for children engaging in resistance training. We were interested in whether such scales could be used effectively to monitor resistance exercise global exertion in overweight and obese children, a population that deserves urgent research attention with regard to exercise. Resistance exercise has been previously shown to be well tolerated by children $(3,20)$. Obese children who have undertaken resistance training programs have significantly increased their lean body mass and bone mineral content (20).

By using the session RPE method we wanted to know whether a single postexercise global rating of exertion experienced during the exercise session would provide essentially the same information as taking RPE measures throughout the entire session. Therefore the purpose of this study was to investigate the use of the children's OMNI-RES scale for monitoring the intensity of different modes of resistance training by comparing values obtained after every set of exercise and after the whole training session (session RPE).

\section{Methods}

\section{Participants}

The participants in this study were 61 children ( 28 girls, 33 boys; mean age $=9.7$ \pm 1.4 years, height $1.47 \pm 0.08 \mathrm{~m}$, weight $55.3 \pm 10.8 \mathrm{~kg}$, BMI $25.6 \pm 3.4$ ). The children had no experience with resistance training before participating in this study. All the children were classified as overweight or obese according to body mass index (BMI). Children with a BMI 95th percentile were classified as obese, and those with a BMI 85th percentile were classified as overweight (8). Maturity status was self-reported (Tanner stage) by participants with the help of their parent(s). Forty-six children were in Tanner Stage 1 and 15 were in Stage 2. The study was approved by the Edith Cowan University Human Research Ethics Committee. All children and their legal guardians were informed of the experimental risks and signed a consent document before the start of the study. 


\section{Experimental Procedures}

The resistance training sessions consisted of total body workouts using a combination of different body weight and power exercises, as well as a variety of equipment (Table 1). The order of the exercises was kept consistent for all children. The primary aim of the exercise program was to incorporate exercises that required minimal equipment including dumbbells, elastic bands, medicine balls and weighted bags so as to assess the program for effectiveness with maximum transference to a wide range of settings such as schools and health-fitness settings. The program consisted of varying training loads within each week of training (i.e., undulating variation). This type of program design has previously been found to be effective for improving body composition (increasing lean body mass and decreasing body fat) in normal weight boys $(19,3)$. These programs have included specific days targeting strength, power or hypertrophy and utilizing different repetitions, sets and rest periods between sets.

Table 1 Training Program

\begin{tabular}{cclccccc}
\hline Day & Order & Exercise & Sets & Min Reps & Max Reps & Tempo & Rest \\
\hline 1 & 1 & Squat $\left(90^{\circ}\right)$ & 3 & 8 & 10 & Controlled & $90 \mathrm{~s}$ \\
& 2 & Press & 3 & 8 & 10 & Controlled & $90 \mathrm{~s}$ \\
& 3 & Upright row & 3 & 8 & 10 & Controlled & $90 \mathrm{~s}$ \\
& 4 & Lunge & 3 & 8 & 10 & Controlled & $90 \mathrm{~s}$ \\
& 5 & Push-Up & 3 & & Max & Controlled & $90 \mathrm{~s}$ \\
& 6 & Arm curl & 3 & 8 & 10 & Controlled & $90 \mathrm{~s}$ \\
& 7 & Tricep dip & 3 & 8 & 10 & Controlled & $90 \mathrm{~s}$ \\
& 8 & Abdominal & 3 & 12 & 15 & Controlled & $90 \mathrm{~s}$ \\
\hline 2 & 1 & Squat $\left(90^{\circ}\right)$ & 3 & 10 & 12 & Controlled & $60 \mathrm{~s}$ \\
& 2 & Deadlift & 3 & 10 & 12 & Controlled & $60 \mathrm{~s}$ \\
& 3 & Chest fly & 3 & 10 & 12 & Controlled & $60 \mathrm{~s}$ \\
& 4 & Front raise & 3 & 10 & 12 & Controlled & $60 \mathrm{~s}$ \\
& 5 & Row & 3 & 10 & 12 & Controlled & $60 \mathrm{~s}$ \\
& 6 & Calf raise & 3 & 12 & 15 & Controlled & $60 \mathrm{~s}$ \\
& 7 & Tricep extensions & 3 & 10 & 12 & Controlled & $60 \mathrm{~s}$ \\
& 8 & Abdominal & 3 & 12 & 15 & Controlled & $60 \mathrm{~s}$ \\
\hline 3 & 1 & CMJ & 3 & 5 & 5 & Fast & $3 \mathrm{mins}$ \\
& 2 & High pull & 3 & 3 & 3 & Fast & $3 \mathrm{mins}$ \\
& 3 & Press & 3 & 5 & 5 & Fast & $3 \mathrm{mins}$ \\
& 4 & Upright row & 3 & 5 & 5 & Fast & $3 \mathrm{mins}$ \\
& 5 & SJ & 3 & 3 & 3 & Fast & $3 \mathrm{mins}$ \\
& 6 & Lying press & 3 & 5 & 5 & Fast & $3 \mathrm{mins}$ \\
& 7 & Broad jump & 3 & 3 & 3 & Fast & $3 \mathrm{mins}$ \\
& 8 & Abdominal & 3 & 10 & 10 & Fast & $60 \mathrm{~s}$ \\
\hline
\end{tabular}

Note. $\mathrm{CMJ}=$ countermovement jump; $\mathrm{SJ}=$ static jump (performed with $2-3 \mathrm{~s}$ hold at the bottom position). 
Before beginning the program, all participants were familiarized with the exercise techniques in a separate session. The first workout of the week consisted of 3 sets of 8-10 repetition maximum (RM) with $90 \mathrm{~s}$ rest between sets. The second workout of the week involved high-volume, moderate-intensity training sessions performed using 10-12 RM with one minute rest between sets. The third workout of the week included predominantly explosive power exercises using 3 sets of 3-5 repetitions with $3 \mathrm{~min}$ rest between sets. Loads were used that could be tolerated by the participants and the sets were not performed to volitional fatigue. Each session was supervised by qualified exercise instructors who kept detailed training logs of all the sets, repetitions and exercises performed by each participant. For each session, the children performed a 5 min warm-up (consisting of running, jumping and dynamic exercise) and a 5-10 min cool-down (consisting of static stretching).

\section{Perceived Exertion Scale}

To assess average RPE during the exercise sessions, standard instructions and anchoring procedures were explained during the familiarization sessions (2). Participants were asked to rate their perceived exertion following the completion of each lifting set based on the OMNI-RES scale (Figure 1). A series of anchoring tests was used to establish high and low perceptual anchors (15). Approximately 10-15 min following each training session, session RPE data were collected by participants rating their perceived exertion based on the OMNI-RES scale by answering the following question, "How was your workout?" (6). Previous research has shown that this is the minimal time required to obtain an accurate reflection of the global intensity of the exercise session (17). The participants would verbally indicate a number after looking at the scale and this was designed to give an indication of how hard the exertion felt during the exercise session. Numbers from 0 to 10 on the scale were used to rate the intensity of the entire workout session (15). A rating of 0 was associated with rest and the highest rating of 10 referred to maximal effort. This rating of the perceived exertion of the whole session was different from the more standard approach that asks a participant to rate the exertion associated with a particular exercise or set. The aim of the session RPE was to provide a rating of the perceived exertion for the overall resistance training sessions. The OMNI-RES was in full view of the participants at all times during the sessions. In addition, a RPE was obtained using the OMNI-RES scale following each set during the exercise sessions. These values were used to calculate the average RPE of the session.

A pilot study was performed during the familiarization sessions for the exercises to determine which scale to use; the four picture OMNI-RES (15) or the five picture scale developed by Faigenbaum et al. (4). We found that the children were able to understand the verbal expressions and how they were interpreted numerically when using the four-picture scale. To confirm this, we calculated the reliability of the session RPE measures over two sessions using the same exercises and loads in 10 participants, and reliability results are presented below. We chose to use an undifferentiated rating for the overall body (15), for both the within session measures and the session RPE, as opposed to a differentiated rating for the active muscles, as this was easier for the children to understand. 


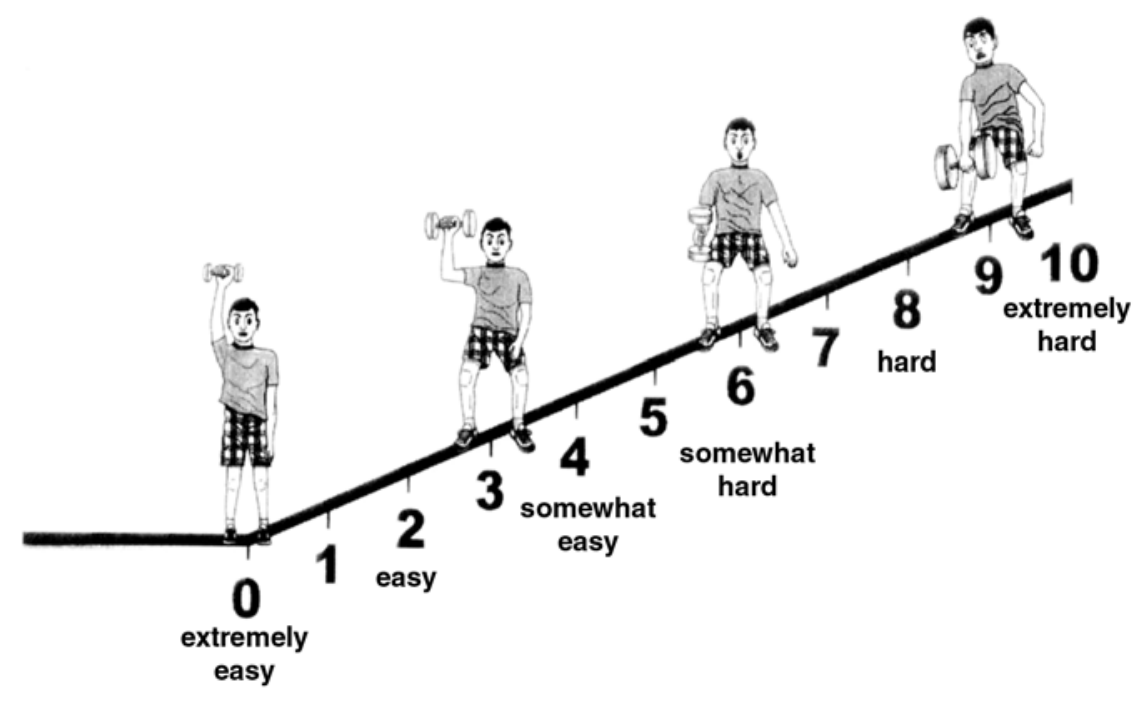

Figure 1 - OMNI-RES scale for children.

\section{Statistical Analyses}

A two-way within-participants repeated measures analysis of variance (ANOVA) was used to test for differences in RPE over time. Each participant's mean RPE values from the sets of each exercise during the session were averaged and compared with their session RPE rating. Averages of each exercise were also compared with the session RPE to identify any significant differences.

Correlations between the variables were calculated using the Pearson product moment correlation coefficient. The magnitude of the differences between values was also interpreted using Cohen's Effect Size (ES). Reliability of the session RPE measures was determined by calculating intraclass correlation coefficients, typical error, and typical error as a coefficient of variation (CV). The criterion for statistical significance was set at $p \leq .05$.

\section{Results}

There was a significant difference between average RPE $(1.68 \pm 0.61)$ and Session RPE $(3.10 \pm 1.18)$ during the four weeks of training and the effect size was large $(p<.05, \mathrm{ES}=1.37)$. There was no significant change in session RPE over the four weeks of training (Figure 2). There was also no significant change in the average RPE over the four weeks of training (Figure 2). The overall correlation coefficient between mean RPE and session RPE values was significant ( $r=.88, p$ $<.05)$. The reliability of the session RPE was high with $\mathrm{ICC}=0.94$, typical error $=0.49$ and $\mathrm{CV}=7.7 \%$. 


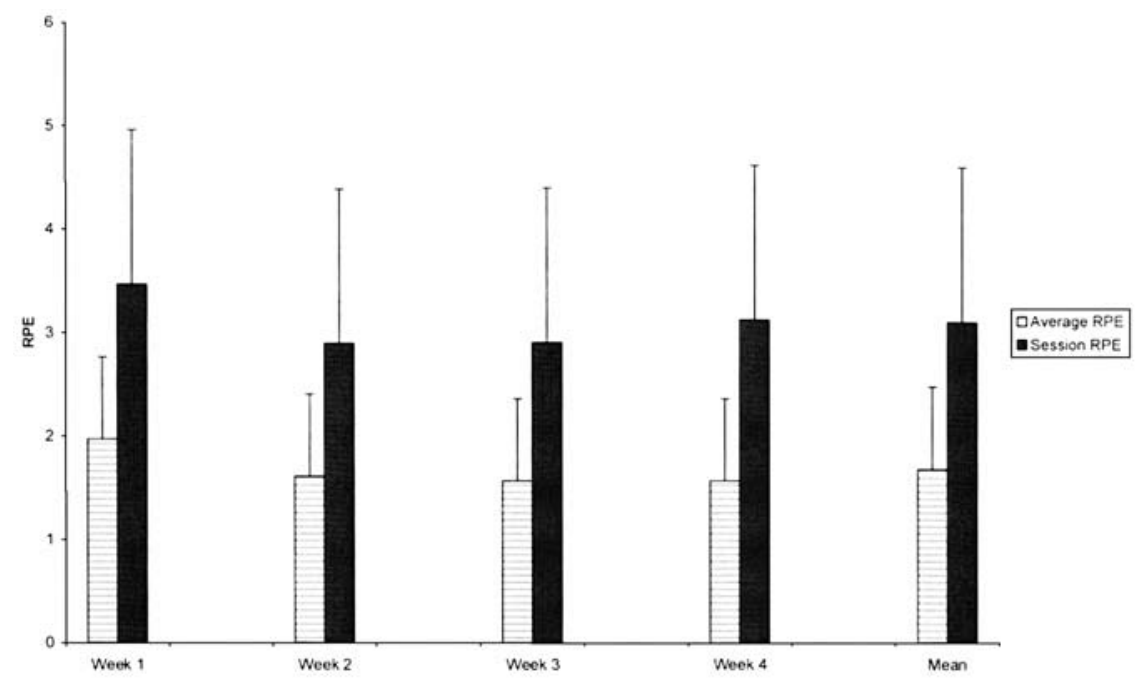

Figure 2 - Average and session RPE values across the 4-week training intervention. Values are mean $\pm S D$.

\section{Discussion}

Previous research has demonstrated the effectiveness of the session RPE method for monitoring training intensity in adults (7) and specifically for resistance exercise $(2,18)$. There is no previous research investigating the efficacy of session RPE in other populations such as children, however. The findings of the current study indicate that the RPE values are higher when OMNI-RES measures are obtained following the whole training session than when expressed as an average for all exercise sets. This difference suggests that in overweight children the session RPE provides different information than the average RPE across the entire session. However, there was a very high correlation between the session and average RPE and the session RPE method was highly reliable.

Because this was the initial portion of a 24-week training program, and these children were unaccustomed to this type of exercise, we did attempt to keep the intensity of the exercise sessions very low. Like other researchers (4), we found in our pilot testing that there is a tendency for children to self-select resistances that exceed their capacity and to be unable to compete the required repetitions. Therefore we ensured that the children were performing the exercises with correct technique rather than focusing on resistance lifted.

A reason why the session RPE rating was significantly higher than average RPE could be that the children included the warm-up and cool-down as part of their rating of the session. RPE values were not obtained for the warm-up and cool-down components or included in the calculation of average RPE. These types of exercises were considered low intensity (stretching, fun games, some running) and were for a short duration (5-10 min), but might still have contributed to the inflation in the 
session RPE compared with the average RPE value. We also asked the children to give the session RPE 15 minutes following the cool-down. Previous work in adult populations suggests that the session RPE should be taken 30 min after the end of a workout so as to avoid particularly difficult or easy elements near the end of the exercise session from distorting the entire rating of the session (17). It might be possible that the cool-down was perceived as being difficult by the participants. We did attempt, however, to keep this to moderate stretching, so this is unlikely. It is possible that the inclusion of static stretching in the cool-down inflated the value because children who are obese might find these exercises difficult because of their increased adiposity, which impacts on their range of motion.

Alternatively, the session RPE could well be reflecting residual fatigue that was not adequately perceived during individual sets of exercise (18). The dominant perceptual signal during resistance training is muscle tension, and not the persistent hypernea that is normal during aerobic exercise. It is quite reasonable to suggest that the relatively less intense exertional signals attributable to low muscular tension during the exercises, together with a delayed sense of muscular effort attributable to microtrauma, could account for the systematically higher ratings for the entire exercise session. This is analogous to the apparent increase in RPE with prolonged exercise at a constant submaximal intensity that is evident in a marathon runner rating a race equal to maximal intensity even though the momentary intensity of running might never have exceed hard intensity (6).

The possibility that participants might have underestimated their perceived exertion at lower intensities agrees with previous research by Faigenbaum et al. (4) who found that their sample of children rated 35\% and 55\% of 1RM as very easy. On average, the participants in the current study rated the intensity from easy to somewhat easy. This did not change over the 4-week training period, which would suggest that lack of practice and instruction with the scale was not the issue. It is more likely a reflection of the lower intensity of the sessions that were used with these participants. We did not measure, however, specific 1RM's for each exercise, so there is no way to confirm this hypothesis, and this is thus an avenue of future research. It is also possible that by emphasizing correct technique we underestimated the RM loads, and this might also go some way to explaining the current findings.

This use of the session RPE method is different from the conventional approach, which asks participants to rate with highly standardized verbal instructions how intense the exertion is perceived to be at that particular moment. The research to date has suggested that session RPE also provides a valid (6) and reliable $(2,13)$, indicator of resistance training intensity in adult populations. In this study, we showed the session RPE to be also reliable in this population of children who were overweight. Its effectiveness across a variety of resistance training intensities and modes of training has been shown $(6,17,18)$. Some studies have also validated pictorial RPE scales, such as the OMNI-RES, in normal weight children (15). Overall, research suggests that these scales can provide information to the practitioner regarding the perceived intensity of resistance exercise. There is also research suggesting RPE can be used to guide exercise prescription and specifically resistance exercise (10), although this is not supported by all research findings (5). Children who are overweight appear to be able to understand and interpret pictorial based RPE scales such as the OMNI-RES. Whether the session RPE can be used 
for more prescriptive purposes with overweight children appears to be an issue worth addressing for researchers.

Additional research is needed to confirm the efficacy of the session RPE in younger populations across a range of exercise modes (e.g., aerobic, interval). We did find it necessary to regularly remind the children about the meaning of the scale. This is similar to what we have found with exercise technique in this population in that children need to be continually taught correct technique and supervised closely at all times. The results of the current study would suggest that there is a high correlation between session RPE and average RPE obtained throughout the exercise session in overweight and obese children, and that the session RPE is a reliable measure. Practitioners should be careful, however, to adequately familiarize children with the OMNI-RES scale and provide appropriate anchoring procedures to ensure its efficacy.

\section{Acknowledgments}

This study was funded by the Telstra Foundation. The authors wish to thank the dedicated group of trainers and the children and parents involved in the study.

\section{References}

1. Cohen, J. Statistical power analysis for the behavioural sciences, 2nd ed. New York: Academic Press, 1988.

2. Day, M.L., M.R. McGuigan, G. Brice, and C. Foster. Monitoring exercise intensity during resistance training using the session RPE scale. J. Strength Cond. Res. 18:353-358, 2004.

3. Faigenbaum, A.D., R.L. Loud, J. O'Connell, S. Glover, J. O'Connell, and W.L. Westcott. Effects of different resistance training protocols on upper-body strength and endurance development in children. J. Strength Cond. Res. 15:459-465, 2001.

4. Faigenbaum, A.D., L.A. Milliken, G. Cloutier, and W.L. Westcott. Perceived exertion during resistance exercise by children. Percept. Mot. Skills. 98:627-637, 2004.

5. Focht, B.C. Perceived exertion and training load during self-selected and imposedintensity resistance exercise in untrained women. J. Strength Cond. Res. 21:183-187, 2007.

6. Foster, C., J.A. Florhaug, J. Franklin, et al. A new approach to monitoring exercise training. J. Strength Cond. Res. 15:109-115, 2001.

7. Foster, C., L.L. Hector, R. Welsh, M. Schrager, M.A. Green, and A.C. Snyder. Effects of specific versus cross-training on running performance. Eur. J. Appl. Physiol. Occup. Physiol. 70:367-372, 1996.

8. Freedman, D., C. Ogden, G. Berenson, and M. Horlick. Body mass index and body fatness in childhood. Curr. Opin. Clin. Nutr. Metab. Care. 8:618-623, 2005.

9. Hopkins, W.G. A new view of statistics. Available at: http://www.sportsci.org/resource/ stats/index.html. Accessed, 2007.

10. Lagally, K.M., and A.J. Amorose. The validity of using prior ratings of perceive exertion to regulate resistance exercise intensity. Percept. Mot. Skills. 104:534-542, 2007.

11. Lagally, K.M., S.T. McCaw, G.T. Young, H.C. Medema, and D.Q. Thomas. Ratings of perceived exertion and muscle activity during the bench press exercise in recreational and novice lifters. J. Strength Cond. Res. 18:359-364, 2004. 
12. Lagally, K.M., R.J. Robertson, K.I. Gallagher, et al. Perceived exertion, electromyography, and blood lactate during acute bouts of resistance exercise. Med. Sci. Sports Exerc. 34:552-559, 2002.

13. McGuigan, M.R., A.D. Egan, and C. Foster. Salivary cortisol responses and perceived exertion during high intensity and low intensity bouts of resistance exercise. J. Sports Sci. Med. 3:8-15, 2004.

14. Noble, B.J., and R. Roberston. Perceived exertion, 1st ed. Champaign, IL: Human Kinetics, 1996.

15. Robertson, R.J., F.L. Goss, J.L. Andreacci, et al. Validation of the Children's OMNIResistance Exercise Scale of perceived exertion. Med. Sci. Sports Exerc. 37:819-826, 2005.

16. Robertson, R.J., F.L. Goss, J.A. Bell, et al. Self-regulated cycling using the Children's OMNI Scale of Perceived Exertion. Med. Sci. Sports Exerc. 34:1168-1175, 2002.

17. Singh, F., C. Foster, D. Tod, and M.R. McGuigan. Monitoring different types of resistance training using session rating of perceived exertion. Int. J. Sport Phys. Perf. 2:34-45, 2007.

18. Sweet, T.W., C. Foster, M.R. McGuigan, and G. Brice. Quantitation of resistance training using the session rating of perceived exertion method. J. Strength Cond. Res. 18:796-802, 2004.

19. Volek, J.S., A.L. Gómez, T.P. Scheett, et al. Increasing fluid milk favorably affects bone mineral density responses to resistance training In adolescent boys. J. Am. Diet. Assoc. 103:1353-1356, 2003.

20. Yu, C., R. Sung, R. So, et al. Effects of strength training on body composition and bone mineral content in children who are obese. J. Strength Cond. Res. 19:667-672, 2005. 
Copyright of Pediatric Exercise Science is the property of Human Kinetics Publishers. Inc. and its content may not be copied or emailed to multiple sites or posted to a listserv without the copyright holder's express written permission. However, users may print, download, or email articles for individual use. 